\title{
Diastolic abnormalities in systemic sclerosis: evidence for associated defective cardiac functional reserve
}

Gabriele Valentini, Dino Franco Vitale, Anna Giunta, Stefania Maione, Giusto Gerundo, Mariarosaria Arnese, Enrico Tirri, Nicola Pelaggi, Attilio Giacummo, Giuseppe Tirri, Mario Condorelli

\begin{abstract}
Objective-To investigate the pattern of diastolic abnormalities in patients with systemic sclerosis (SSc) and the relationship between impaired ventricular filling and systolic function.

Methods-Twenty four patients with SSc underwent $M$-mode and two dimensional echocardiography using echo-Doppler and gated blood pool cardiac angiography, both at rest and after exercise.

Results-An impaired diastolic relaxation of the left ventricle was detected in 10 of the 24 patients with SSc. Left ventricular ejection fraction at rest in these 10 patients with impaired ventricular filling did not differ from that in the remaining 14 patients, but eight of the 10 failed to increase their ejection fraction during exercise, compared with two of the 14 with normal ventricular filling $(p=0.003)$.

Conclusion-Impaired relaxation of the left ventricle is a recently described feature of scleroderma heart disease. Diastolic dysfunction in SSc could depend on myocardial fibrosis or myocardial ischaemia, or both. It was found to be associated with a defective cardiac functional reserve. However, its prognostic significance remains to be clarified.
\end{abstract}

(Ann Rheum Dis 1996; 55: 455-460)

Myocardial fibrosis is considered to be a pathological hallmark of myocardial disease in systemic sclerosis (SSc). ${ }^{1-3}$ Its pathogenesis has not been elucidated completely, but vasoconstriction of small intramyocardial arteries is likely to be a contributing factor. ${ }^{4-8}$ Both myocardial fibrosis and myocardial ischaemia are known to affect ventricular filling, ${ }^{9} 10$ and we have observed impaired ventricular filling in a significant percentage of patients with SSc, in the absence of any clinically evident myocardial disease, systolic dysfunction, or other condition known to affect diastolic function. ${ }^{11}$ Others have reported similar results from either echocardiography or angiography. ${ }^{12} 13$ However, the clinical significance of impaired ventricular filling remains to be determined.

In the present study, we investigated 24 patients with SSc for diastolic and systolic heart function, using both echocardiography and gated blood pool cardiac scintigraphy.

\section{Subjects and methods}

PATIENTS

From 1 January 1993 to 31 December 1994, 75 patients with SSc (67 women and eight men, aged 26 to 73 years) all of whom satisfied the American College of Rheumatology (formerly the American Rheumatology Association) preliminary criteria for the classification of $\mathrm{SSc}^{14}$ were admitted to the Division of Rheumatology of the Second University of Naples. They were all examined carefully in order to define the subset of their disease according to Giordano et $a l,{ }^{15}$ the extent and the degree of skin sclerosis according to Steen et $a l,{ }^{16}$ and the severity of internal organ involvement, which was scored according to Casas et al. ${ }^{17}$ All the patients underwent chest radiography, electrocardiography (ECG) at rest, M-mode echocardiography, and pulmonary function tests including the evaluation of diffusing capacity for carbon monoxide.

Among the 75 patients, 25 were found to be affected with conditions known to impair ventricular filling: congestive heart failure, pericardial effusions, coronary artery disease, and arterial hypertension with left ventricular hypertrophy. The remaining 50 patients, including four hypertensive patients without increased left ventricular wall thickness $(<13 \mathrm{~mm}$ ) were invited to undergo bicycle exercise and 24 hour Holter ECG, two dimensional echocardiography with Doppler examination, and gated blood pool scintigraphy. Twenty four of them gave their informed consent to participate: 20 had no overt evidence of heart involvement as detected by basal investigations (ECG at rest and $\mathrm{M}$-mode echocardiography), and four had arterial hypertension without left ventricular hypertrophy.

All the patients had been treated with calcium channel blockers, which were withdrawn seven days before the echo-Doppler and radionuclide cardiac angiography studies were performed.

Following our previous study, ${ }^{11}$ we subdivided the patients with SSc into two groups: those with (group $\mathbf{A} ; \mathbf{n}=10$ ) and those without (group $B ; n=14$ ) altered ventricular filling as defined by inversion of the transmitral echo-Doppler flow profile (E:A ratio). 


\section{CONTROLS}

During the same period, patients admitted to the clinic with either osteoarthritis or fibrositis were invited to take part in the study. Thirty four gave their informed consent, and 24 of these showed no evidence of cardiovascular disease as detected by history, clinical examination, ECG at rest, or echocardiography, and were recruited to the study. Because the 10 group A patients with SSc and an inverted E:A ratio proved to be older than the 14 group $B$ patients with normal ventricular filling (55 (SD 9) years compared with 42 (7) years; $p<0.05$ ), we subdivided the control subjects into two subsets strictly matched with the patients for gender and age: subset I $(\mathrm{n}=10)$ aged 54 (7) years, and subset II $(n=14)$ aged $42(8)$ years.

\section{ECHOCARDIOGRAPHIC AND DOPPLER} EXAMINATION

M-mode and two dimensional echocardiographic examinations were performed with the patient in a partial left lateral decubitus position, during a quiet and expiratory breath holding state, in a standard manner by means of an HP $77020 \mathrm{AC}$ ultrasound imager, using a 3.5 or $2.5 \mathrm{MHz}$ transducer. The parasternal short axis at the mid-ventricular level was used to derive left ventricular end-diastolic dimensions and left ventricular wall thickness, according to the American Society of Echocardiography recommendations. ${ }^{18}$ Ejection fraction (ellipse biplane method) was calculated as an index of left ventricular systolic pump function. In addition, a complete pulsed and continuous wave Doppler examination was performed in a standard manner. ${ }^{11}$ The following indices of left ventricular filling were considered: peak flow velocity at early (peak E) and late (peak A) diastole, their ratio (E:A), deceleration time (DT) (defined as the time interval required for the $\mathrm{E}$ velocity to decline from its peak to the baseline), and isovolumic relaxation time (IRT) (time elapsing from aortic valve closure to the beginning of transmitral flow). Echocardiographic images were stored on videotape; all measurements were analysed manually and results were expressed as the mean of at least three consecutive cardiac cycles.

Intraobserver variability in the assessment of the Doppler echocardiographic measurements was determined by measuring 10 cardiac cycles on the ventricular inflow velocity trace before and after the radionuclide study. The determination of interobserver variability was based on analysis of the same sets of cardiac cycles by an independent observer. The intraobserver and interobserver coefficients of variation were less than $4.0 \%$ and $4.5 \%$, respectively.

GATED BLOOD POOL CARDIAC SCINTIGRAPHY Radionuclide cineangiography was performed at rest with the patient in the supine position, using a standard technique. ${ }^{19}$ High temporal resolution (10-20 ms/frame) left ventricular time activity curves were generated from the cardiac image sequence by computer based ECG gating in order to assess diastolic function accurately. Left ventricular timeactivity curves were generated from the cardiac image sequence, after background correction with a single fixed left ventricular region of interest, without spatial or temporal smoothing. Indices of global left ventricular function were derived by computer analysis of the background corrected time-activity curve. Ejection fraction and peak ejection rate (PER) were evaluated as measures of left ventricular systolic function. Peak filling rate (PFR) and the contribution of atrial systole to left ventricular filling volume (LVVa) were evaluated to investigate left ventricular filling. PFR was normalised for both end-diastolic counts and stroke counts, the latter in order to avoid any influence of ejection fraction variation on PFR. LVVa was determined as the ratio between the contribution of atrial systole to left ventricular filling and the diastolic volume. ${ }^{19}$ Finally, the PFR:PER ratio (F:E ratio) was evaluated.

Studies were conducted at rest and during maximal supine exercise performed with a bicycle ergometer. Exercise loads were increased by $25 \mathrm{~W}$ every two minutes until the development of fatigue, limiting dyspnoea, angina, or ventricular arrhythmias. Heart rate and blood pressure were monitored during exercise. Radionuclide normal resting left ventricular function was defined by an ejection fraction value within a range defined by $\pm 2 S D$ from the mean of the 24 controls (mean 55 (SD 7.5)\%). A normal response to exercise was defined as an increase in ejection fraction of $5 \%$ or more with respect to the baseline value.

STATISTICAL ANALYSIS

All values are expressed as mean (SD). Student's $t$ test, Mann-Whitney $U$ test, and Fisher's exact test were used to analyse the data.

\section{Results}

Among the 24 patients (22 women and two men, aged 32-73 years (median 45.5)), 11 were affected with limited cutaneous SSc (lcSSc) (scleroderma confined to the fingers), four had intermediate cutaneous SSc (icSSc) (scleroderma involving the limbs and face), and nine had diffuse cutaneous SSc (dcSSc) (scleroderma involving the trunk). Their disease duration was from two to 39 years (median 10.5).

Four patients were affected with arterial hypertension that was controlled by angiotensin converting enzyme inhibitors. In two of them the onset of arterial hypertension had occurred before the first symptom of SSc; in the third, arterial hypertension had appeared three years after the onset of SSc, but was not associated with any finding of altered renal function. The fourth patient had survived a scleroderma renal crisis two years before the time of the study. In one of the four, coronary 
Table 1 Main anatomical parameters and ejection. fraction (EF) values detected by echocardiography in 24 patients with SSc and 24 controls

\begin{tabular}{lcc}
\hline & SSc patients & Controls \\
\hline LVIDd (mm) & $46 \cdot 1(4 \cdot 7)$ & $47 \cdot 9(8)$ \\
IVSth (mm) & $8 \cdot 2(1 \cdot 7)$ & $8 \cdot 9(2 \cdot 1)$ \\
PWth (mm) & $8 \cdot 2(1 \cdot 5)$ & $8 \cdot 1(1 \cdot 5)$ \\
LAD (mm) & $33 \cdot 1(4)$ & $32 \cdot 5(3 \cdot 4)$ \\
EF $(\%)$ & $55 \cdot 1(4 \cdot 2)$ & $57 \cdot 5(3 \cdot 5)$ \\
\hline
\end{tabular}

Values are mean (SD).

LVIDd = Left ventricular internal diameter in diastole IVSth = interventricular septal thickness; PWth = posterior wall thickness; LAD = left atrium diameter.

No significant differences between groups (Student's $t$ test).

Table 2 Resting echo-Doppler and radionuclide indexes of left ventricular filling in 24 patients with SSc and 24 gender and age matched controls

\begin{tabular}{lccc}
\hline & SSc patients & Controls & $p \dagger$ \\
\hline Peak E (cm/s) & $59 \cdot 2(15 \cdot 7)$ & $65 \cdot 3(8)$ & $>0 \cdot 05$ \\
Peak A (cm/s) & $56 \cdot 2(19 \cdot 9)$ & $36 \cdot 7(4 \cdot 9)$ & 0.0001 \\
IRT (ms) & $96 \cdot 2(24 \cdot 1)$ & $71 \cdot 7(6 \cdot 2)$ & 0.0001 \\
DT (ms) & $162 \cdot 7(34)$ & $148 \cdot 5(9 \cdot 8)$ & 0.059 \\
PFR (SV/s) & $5 \cdot 4(1 \cdot 4)$ & $5.3(0 \cdot 7)$ & $>0.05$ \\
LVVa (\%) & $9 \cdot 5(4 \cdot 6)$ & $3.9(2 \cdot 0)$ & 0.0001 \\
F:E ratio & $1 \cdot 1(0 \cdot 3)$ & $1 \cdot 1(0 \cdot 2)$ & $>0.05$ \\
\hline
\end{tabular}

Values are mean (SD).

Peak E, Peak $A=$ peak flow velocity at early $(E)$ and late $(A)$ diastole; IRT = isovolumic relaxation time; $\mathrm{DT}=$ deceleration time; $\mathrm{PFR}_{\mathrm{Sv}}=$ peak filling rate corrected for stroke volume; LVVa = contribution of atrial systole to left ventricular filling volume; F:E ratio = ratio of peak filling rate to peak ejection rate.

†Mann-Whitney $U$ test.

artery disease was also detected by exercise ECG.

Table 1 shows the values of the main anatomical parameters and of left ventricular ejection fraction determined by echocardiography in the 24 patients with SSc and in the 24 controls. None of the patients, including the four with arterial hypertension, was found to have cardiac hypertrophy.

Table 2 shows resting echo-Doppler indices and radionuclide ventriculographic variables in the 24 controls and in the 24 patients with SSc. A faster Apeak, a longer IRT, and an increased LVVa were found in the 24 patients with SSc compared with controls. In addition, a slower Epeak and a longer Tdec were present, but their differences between the groups did not reach statistical significance.

When we compared group A (patients with SSc and an inverted E:A ratio) with the age matched subset I of control subjects, a statistically significant difference was detected for all the echo-Doppler and cardiac angiography parameters of left ventricular

Table 3 Echo-Doppler and radionuclide indexes of left ventricular filling in patients with $S S c$, with (group $A$ ) and without (group B) inverted $E: A$ ratio, and in their respective gender and age matched controls (subset I and subset II)

\begin{tabular}{|c|c|c|c|c|c|c|}
\hline & $\begin{array}{l}\text { Group } A \\
\text { SSc patients } \\
(n=10)\end{array}$ & $\begin{array}{l}\text { Subset I } \\
\text { Controls } \\
(n=10)\end{array}$ & $p \dagger$ & $\begin{array}{l}\text { Group B } \\
\text { SSc patients } \\
(n=14)\end{array}$ & $\begin{array}{l}\text { Subset II } \\
\text { Controls } \\
(n=14)\end{array}$ & $p \dagger$ \\
\hline $\begin{array}{l}\text { Peak E }(\mathrm{cm} / \mathrm{s}) \\
\text { Peak A (cm/s) } \\
\text { IRT (ms) } \\
\text { DT (ms) } \\
\text { PFR }(\mathrm{SV} / \mathrm{s}) \\
\text { LVVa (\%) } \\
\text { F:E ratio }\end{array}$ & $\begin{array}{c}50 \cdot 5(14 \cdot 7) \\
69(24 \cdot 1) \\
105.5(30 \cdot 5) \\
164.5(41.5) \\
4.3(0 \cdot 7) \\
13.3(3 \cdot 8) \\
0.9(0.2)\end{array}$ & $\begin{array}{c}67 \cdot 1(7.8) \\
36 \cdot 8(5 \cdot 6) \\
71 \cdot 2(5 \cdot 6) \\
148(8 \cdot 2) \\
5 \cdot 1(0 \cdot 3) \\
3(1 \cdot 9) \\
1(0 \cdot 1)\end{array}$ & $\begin{array}{l}0.01 \\
0.0001 \\
0.001 \\
>0.05 \\
0.01 \\
0.0001 \\
>0.05\end{array}$ & $\begin{array}{c}65 \cdot 3(13 \cdot 7) \\
47 \cdot 1(9 \cdot 1) \\
83 \cdot 7(26 \cdot 3) \\
161 \cdot 4(27 \cdot 5) \\
6 \cdot 1(1 \cdot 4) \\
6 \cdot 8(2 \cdot 8) \\
1 \cdot 2(0 \cdot 2)\end{array}$ & $\begin{array}{c}63.9(8 \cdot 1) \\
36 \cdot 7(4 \cdot 6) \\
72 \cdot 1(6 \cdot 8) \\
148 \cdot 9(11 \cdot 1) \\
5.6(0.9) \\
4.5(1 \cdot 8) \\
1 \cdot 2(0 \cdot 2)\end{array}$ & $\begin{array}{l}>0.05 \\
0.003 \\
0.005 \\
>0.05 \\
>0.05 \\
0.03 \\
>0.05\end{array}$ \\
\hline
\end{tabular}

Values are mean $(\mathrm{SD})$

Peak E, Peak A = peak flow velocity at early (E) and late (A) diastole; IRT = isovolumic relaxation time; $\mathbf{D T}=$ deceleration time; $\mathbf{P F R}_{\mathrm{Sv}}=$ peak filling rate corrected for stroke volume; $\mathrm{LVVa}=$ contribution of atrial systole to left ventricular filling volume; $F: E$ ratio = ratio of peak filling rate to peak ejection rate.

tMann-Whitney $U$ test.
Table 4 Echo-Doppler and radionuclide indexes of left ventricular filling in six normotensive patients with $S S c$ with inverted $E: A$ ratio, and in their respective age matched controls

\begin{tabular}{|c|c|c|c|}
\hline & SSc patients & Controls & $p \dagger$ \\
\hline Peak E $(\mathrm{cm} / \mathrm{s})$ & $46 \cdot 8(11 \cdot 8)$ & $67.5(9 \cdot 6)$ & 0.01 \\
\hline Peak A $(\mathrm{cm} / \mathrm{s})$ & $61.2(14.9)$ & $35.6(5.6)$ & 0.004 \\
\hline IRT (ms) & $90.8(16.8)$ & $70 \cdot 6(6 \cdot 7)$ & 0.02 \\
\hline DT (ms) & $155.8(15 \cdot 6)$ & $145.8(5.8)$ & $>0.05$ \\
\hline $\mathrm{PFR}_{\mathrm{SV}}(\mathrm{SV} / \mathrm{s})$ & $4.5(0 \cdot 8)$ & $5 \cdot 1(0 \cdot 3)$ & 0.08 \\
\hline LVVa (\%) & $13 \cdot 1(4 \cdot 3)$ & $2 \cdot 2(0 \cdot 6)$ & 0.004 \\
\hline $\mathrm{F}: \mathrm{E}$ ratio & $0.9(0.2)$ & $1 \cdot 1(0 \cdot 1)$ & $>0.05$ \\
\hline
\end{tabular}

Values are mean $(\mathrm{SD})$.

Peak E, Peak $A=$ peak flow velocity at early $(E)$ and late (A) diastole; IRT = isovolumic relaxation time; $\mathrm{DT}=$ deceleration time; $\mathrm{PFR}_{\mathrm{Sv}}=$ peak filling rate corrected for stroke volume LVVa = contribution of atrial systole to left ventricular filling volume; $F: E$ ratio = ratio of peak filling rate to peak ejection rate. †Mann-Whitney $U$ test.

filling except for DT and F:E ratio. Comparing group B (patients with SSc but normal ventricular filling) and the age matched subset II of controls, a significant difference was detected for peak A, IRT, and LVVa-the same parameters that differed for the whole series of patients with SSc compared with the entire group of controls (table 3 ).

When the six normotensive patients with SSc and an inverted E:A ratio were compared with their respective six controls matched for gender and age, significant differences emerged for all the parameters investigated, except for F:E ratio and DT (table 4).

Among the 10 patients with an inverted E:A ratio, five were affected with dcSSc and five with either icSSc or lcSSc. The prevalence of an inverted E:A ratio in patients with dcSSc (five of nine) did not differ significantly from that in patients with icSSc or lcSSc (four of 15) $(p>0.05)$. No other correlation emerged with any other epidemiological or clinical feature, including the presence and severity of lung involvement.

\section{LEFT VENTRICULAR SYSTOLIC FUNCTION AT} REST

At rest, there were no statistically significant differences between the two groups of patients with regard to the scintigraphic variables of left ventricular systolic function. In particular, ejection fraction values $(44 \cdot 1(7 \cdot 8) \%$ in group A compared with $44 \cdot 1(6 \cdot 7) \%$ in group B), and PER normalised for either end-diastolic volume $\left(\mathrm{PER}_{\mathrm{EDV}}\right)$ or stroke volume $\left(\mathrm{PER}_{\mathrm{SV}}\right)$ did not differ between the two groups of patients $\left(\mathrm{PER}_{\mathrm{EDV}} 1.9(0.3) \mathrm{EDV} / \mathrm{s}\right.$ in group $\mathrm{A}$ compared with $2 \cdot 0(0 \cdot 27) \mathrm{EDV} / \mathrm{s}$ in group $\mathrm{B}$; $\mathrm{PER}_{\mathrm{SV}} 4 \cdot 7(0 \cdot 8) \mathrm{SV} / \mathrm{s}$ in group A compared with $5 \cdot 2(1 \cdot 3) \mathrm{SV} / \mathrm{s}$ in group B).

It is worth noting that ejection fraction was assessed using a single left ventricular area of interest, accounting for ejection fraction values smaller than those detected using a variable region of interest.

EXERCISE RADIONUCLIDE ANGIOGRAPHY In patients studied during maximal supine exercise, exercise was terminated in every patient because of fatigue. No dyspnoea or angina occurred. Ischaemic ST changes on the 


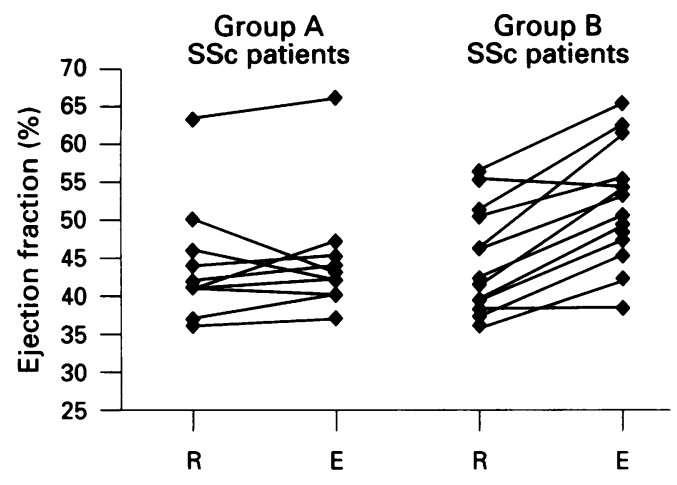

Radionuclide left ventricular ejection fraction at rest $(R)$ and after peak exercise $(E)$ in 10 patients with $S S c$ with (group $A$ ) and in 14 patients with SSc without (group B) resting inverted $E: A$ ratio on echo-Doppler.

ECG were detected in only one patient. A statistically significant difference was detected in mean values of the ejection fraction at peak exercise between the two subgroups of the patients with SSc (figure): in the 10 patients with resting impaired left ventricular filling, ejection fraction at exercise did not differ from resting values $(44.6(8) \%$ compared with $44 \cdot 1$ $(7 \cdot 8) \%)$, but in the 14 patients in group $B$ (normal ventricular filling) a significant increase in ejection fraction values was detected during exercise (from $44 \cdot 1(6 \cdot 7) \%$ to $51.6(7 \cdot 7) \% ; p=0.019)$. A normal increase of ejection fraction during exercise $(>5 \%)$ was detected in two of the 10 patients in group $A$ and in one of the six normotensive patients in that group, but each of these percentages was significantly smaller than values in group B patients (12 of the 14 patients showed a normal increase in their ejection fraction) $(p=0.003$ and $p=0.007$, respectively).

Among the 10 patients with SSc who failed to increase their ejection fraction during exercise, six were affected with lcSSc or icSSc, and four had dcSSc; the SSc subsets thus did not differ with regard to ejection fraction response to exercise. Failure to increase ejection fraction during exercise was not found to correlate with any other clinical or epidemiological feature.

No statistically significant differences were detected for exercise time, exercise work load, or rate-pressure product (RPP) mean values between the two groups of patients with SSc. Mean values of RPP in group A patients with resting impaired ventricular filling were slightly greater than the corresponding mean values observed in group B (20884 (5620) in group A compared with 17569 (4189) in group B). This appeared to be related to the greater heart rate achieved by the patients of group $A$ at peak exercise.

\section{Discussion}

In this study, we investigated 24 patients with SSc for the presence of an abnormal ventricular filling by two techniques: Doppler echocardiography and radionuclide angiography. ${ }^{92021}$ Both methods assess diastolic function indirectly; nevertheless, they have been validated in studies in which significant correlations emerged between the results obtained with either Doppler echocardiography or angiography and those obtained with cardiac catheterisation. ${ }^{22} 23$

An inverted E:A ratio was found in 10 of the 24 patients with SSc who were studied (41\%).

The 10 patients with diastolic dysfunction were older than those without such an abnormality. Older age is known to be associated with abnormal ventricular filling, ${ }^{9}$ but in SSc it may be no more than a contributing factor, as a significant difference in diastolic parameters was also detected between the 10 patients with SSc who had diastolic abnormalities and their 10 strictly age matched control counterparts (table 3 ).

Four of the patients with diastolic abnormalities had arterial hypertension without left ventricular hypertrophy. An inverted $\mathrm{E}: \mathrm{A}$ ratio has been reported in patients with arterial hypertension, even in the absence of cardiac hypertrophy. ${ }^{24}$ Nevertheless, when we compared the six normotensive group A patients with SSc with their respective matched controls, significant differences were still detected in peak E, peak A, IRT, and LVVa (table 4). Thus, when present, hypertension, in common with age, seems simply to be. a contributing factor in inducing diastolic abnormalities in SSc.

The abnormal ventricular filling pattern found in SSc (in the 10 patients with an inverted E:A ratio) was characterised by a longer IRT, a reduced early diastolic filling, and an increased atrial filling velocity on echoDoppler, and by a reduced PFR corrected for stroke volume $\left(\mathrm{PFR}_{\mathrm{SV}}\right)$ and an increased $\mathrm{LVVa}$ on radionuclide angiography (table 3 ). That pattern is considered to indicate an impaired diastolic relaxation and has been reported as an early finding in disorders such as primary and secondary left ventricular hypertrophy (including aortic stenosis and hypertensive heart disease) and coronary artery disease. It can arise as the consequence of myocardial ischaemia or myocardial fibrosis. ${ }^{25}$ In fact, myocardial fibrosis, and myocardial ischaemia, are responsible for asynchronous relaxation by inducing a regional heterogeneity in the timing and magnitude of systolic shortening that affects the synchrony and extent of myocardial relaxation, and by producing non-uniform loading conditions throughout the left ventricle at the onset of relaxation. ${ }^{26-30}$

It is worth noting that patients with SSc without an inverted E:A ratio (group B) were also found to differ statistically from controls with regard to peak A, IRT, and LVVa. Such results seem to indicate that changes in left ventricular filling also occur in patients with SSc who have a normal diastolic function as defined by the value of the E:A ratio. Further studies are required to ascertain whether diastolic abnormalities in SSc reflect the presence of myocardial ischaemia, myocardial fibrosis, or both.

SSc myocardial disease has been reported to be more severe in patients with dcSSc. ${ }^{5}$ As far as diastolic abnormalities are concerned, no difference emerged among SSc subsets in our 
series, and there was no correlation between altered ventricular filling and any other clinical or epidemiological aspect except for older age.

When we examined myocardial pump function at rest, we found echocardiographic ejection fraction to be normal in almost all patients with SSc, except two in whom borderline ejection fraction values were measured. However, the prevailing distribution of the values was towards the lower end of the normal range, consistent with the finding of radionuclide ejection fraction values that were also lower in the patients with SSc than the corresponding values registered in controls.

Patients with SSc both with and without abnormal ventricular filling were found to show similar systolic pump function indices at rest (ejection fraction values $44 \cdot 1 \quad(7 \cdot 8) \%$ compared with $44 \cdot 1(6 \cdot 7) \%$, respectively). However, when systolic function was studied during exercise, a notable difference emerged, the group of patients with SSc who had an abnormal diastolic filling pattern being more frequently unable to increase ejection fraction during exercise than were the remaining patients $(p=0.003)$. It should be noted that the exercise time, work load, and RPP were not found to be different, though a trend toward a small increase in the last parameter was detected in the group of patients with resting filling abnormalities.

When analysing the pathophysiological and clinical significance of changes in ventricular filling, it is necessary to differentiate the truly diastolic abnormalities from those resulting from an altered systolic function. This is particularly true for subjects such as our patients with SSc, who were found to present with a relatively lower radionuclide ejection fraction, even though the method used (a single region of interest as opposed to a variable region of interest) may have contributed to that finding of a low ejection fraction. ${ }^{19}$ In this regard, it is worth emphasising that no difference emerged in resting ejection fraction values between patients with SSc with and without altered ventricular filling. Nonetheless, only the former group failed to increase their ejection fraction normally during exercise. We also corrected PFR values for stroke volume, thus excluding any variation of PFR as a result of changes in ejection fraction. Finally, the similar work load capacity exhibited by patients in the two groups allows us to exclude any influence of a different work load capacity on the abnormal ejection fraction response to exercise.

In 1984, Siegel et $a l^{31}$ investigated 10 patients with SSc who had congestive heart failure and detected normal $(n=5)$ to increased $(n=5)$ resting ejection fraction. These authors argued that a reduced left ventricular compliance and the consequent altered ventricular filling were responsible for congestive heart failure in their patients. The detection of diastolic abnormalities in patients with SSc by echo-Doppler or angiographic study, or both, might make it possible to identify those at risk of developing diastolic failure. This could have important implications for treatment, as inotropic agents and diuretics may be detrimental in patients with congestive heart failure as a result of alterations in diastolic function. ${ }^{10}$

In conclusion, we have demonstrated that impaired ventricular relaxation is frequent in patients with SSc and is significantly associated with a defective cardiac functional reserve. However, its prognostic significance remains to be clarified.

1 D'Angelo W A, Fries J, Masi A T, Shulman L E. Pathologic observations in systemic sclerosis (scleroderma). Am Med 1969; 46: 428-40.

2 Bulkley B H, Ridolfi R L, Salyer W L, Hutchins G M Myocardial lesions of progressive systemic sclerosis: a cause of cardiac dysfunction. Circulation 1976; 53: 483-90.

3 Follansbee W P, Miller T R, Curtiss E J, et al. A controlled clinicopathologic study of myocardial fibrosis in systemic clerosis (scleroderma). $\mathcal{F}$ Rheumatol 1990; 17: 656-62.

4 Follansbee $W$ P, Curtiss E J, Medsger T A Jr, et al. Physiologic abnormalities of cardiac function in progressive systemic sclerosis with diffuse scleroderma. $N$ Engl ₹ Med 1984; 310: 142-8.

5 Follansbee W P, Curtiss E J, Medsger T A Jr, Owens G R Steen V D, Rodnan G P. Myocardial function and perfusion in the CREST syndrome variant of progressive perfusion in the CREST syndrome variant of progressive
systemic sclerosis. Exercise radionuclide evaluation and systemic sclerosis. Exercise radionuclide evaluation and comparis

6 Kahan A, Devaux J Y, Amor B, et al. Nifedipine and thallium-201 myocardial perfusion in progressive systemic sclerosis. $N$ Engl f Med 1986; 314: 1397-402.

7 Alexander E L, Firestein G S, Weiss J, et al. Reversible coldinduced abnormalities in myocardial perfusion and function in systemic sclerosis. Ann Intern Med 1986; 105

8 Ellis W N, Baer A N, Robertson R M, Pinens T, Kronenberg $M$ W. Left ventricular dysfunction induced by cold exposure in patients with systemic sclerosis. $\mathrm{Am}$ by cold exposure in patien

9 Labovitz A J, Pearson A C. Evaluation of left ventricular diastolic function: clinical relevance and recent Doppler echocardiographic insights. Am Heart $\mathcal{f} 1987$; 114 836-51.

10 Brutsaert D L, Sys S U, Gillebert T C. Diastolic failure: pathophysiology and therapeutic implications. $\mathcal{F} \mathrm{Am} \mathrm{Coll}$ Cardiol 1993; 22: 318-25.

11 Maione S, Valentini G, Giunta A, et al. Evaluation of cardiac structures and function in systemic sclerosis by Doppler echocardiography. Cardiology 1991; 79: 165-71.

12 Kazzam E, Waldenstrom A, Landelius J, Hallgren $R$ Arvidson A, Caidhal K. Non-invasive assessment of left ventricular diastolic function in patients with systemic ventricular diastolic function in patients
sclerosis. F Intern Med 1990; 228: 183-92.

13 Pace L, Capelli L, Bove E, et al. Left ventricular diastolic function in systemic sclerosis: assessment by radionuclide angiography. $₹$ Nucl Med 1992; 33: 68-72.

14 Subcommittee for Scleroderma Criteria of the American Rheumatism Association Diagnostic and Therapeutic Criteria Committee. Preliminary criteria for classification of systemic sclerosis (scleroderma). Arthritis Rheum 1980 23: 581-90.

15 Giordano M, Valentini G, Migliaresi S, Picillo U, Vatti M. Different antibody patterns and different prognoses in patients with scleroderma with various extent of skin sclerosis. F Rheumatol 1986; 13: 911-6.

16 Steen V D, Medsger T A Jr, Rodnan G P. D-penicillamine therapy in progressive systemic sclerosis (scleroderma) Ann Intern Med 1982; 97: 652-8.

17 Casas J A, Subauste C P, Alarcon G S. A new promising treatment in systemic sclerosis: 5-fluoruracil. Ann Rheum Dis 1987; 46: 763-7.

18 Sahn D J, De Maria A, Kissl S, Wayman A. Recommendations regarding quantitation in M-mode measurements. Circulation $1978 ; 58$. $1072-83$

19 Bonow R O, Vitale D F, Bacharach S L, Maron B J, Green $M$ V. Effect of aging on asynchronous left ventricular $M$ V. Effect of aging on asynchronous left ventricular
regional function and global ventricular filling in normal regional function and global ventricular filling in no

20 Bonow R A, Bacharach S L, Green M V, et al. Impaired left ventricular filling in patients with coronary artery disease: assessment with radionuclide angiography. disease: assessment with
Circulation 1981; 65: 315-23.

21 Polak J F, Kemper A J, Bianco J A, Paris A F, Tow D E. Resting early peak diastolic filling rate: a sensitive index of myocardial dysfunction in patients with coronary artery disease. $\mathcal{F}$ Nucl Med 1982; 23: 471-8.

22 Channer K S, Culling W, Wilde P, Jones J V. Estimation of left ventricular and diastolic pressure by pulsed doppler ultrasound. Lancet 1986; 1: 1005-7.

23 Magorien D J, Shaffer P, Bush C, et al. Hemodynamic correlates for timing intervals, ejection rate and filling rate derived from the radionuclide angiographic volume curve. Am f Cardiol 1984; 53: 567-71. 
24 Gardin J M, Drayer J J, Rohan M K, et al. Doppler evaluation of left ventricular filling in mild and severe hypertension. f Am Coll Cardiol 1986; 7: 187A

25 Bonow $R$ O. Regional left ventricular nonuniformity. Effects on left ventricular diastolic function in ischemic heart disease, hypertrophic cardiomyopathy, and normal heart. Circulation 1990; 81 (suppl III): 54-65.

26 Brutsaert D L, Rademaker F E, Sys U S. Triple control of relaxation: implications in cardiac disease. Circulation 1983; 69: 190-6.

27 Gaasch W H, Blaustein A S, Bing O H L. Asynchronous (segmental early) relaxation of the left ventricle. $\mathcal{F} \mathrm{Am} \mathrm{Coll}$ Cardiol 1985; 5: 891-7.
28 Blaustein A S, Gaasch W H. Myocardial relaxation. VI. Effects of beta-adrenergic tone and asynchrony on $\mathrm{LV}$ relaxation rate. Am $\mathcal{F}$ Physiol 1983; 244: H417-22.

29 Lew YW, Rasmussen C M. Influence of nonuniformity on rate of left ventricular pressure fall in the dog. Am $\mathcal{Y}$ Physiol 1989; 256: H222-32.

30 Takeuchi T, Fujitani $\dot{K}$, Kurogane $\mathrm{K}$, et al. Effects of left ventricular asynchrony on time constant and extrapolated pressure of left ventricular pressure decay in coronary artery disease. $\mathcal{F}$ Am Coll Cardiol 1985; 6: 597-602.

31 Siegel R J, O'Connor B, Mane I, Criley J M. Left ventricular function at rest and during Raynaud's phenomenon in patients with scleroderma. Am Heart $f$ 1984; 108: patients $1469-76$. 